\title{
Roles of Clinician, Patient, and Community Character- istics in the Management of Pediatric Upper Respiratory Tract Infections
}

\author{
Jeffrey P. Yaeger, $M D, M P H^{1}$ \\ Jonathan L. Temte, MD, $P b D^{2}$ \\ Lawrence P. Hanraban, $\mathrm{PbD}, \mathrm{MS}^{2}$ \\ Ana P. Martinez-Donate, $\mathrm{PbD}^{3}$ \\ 'St. Christopher's Hospital for Children, \\ Drexel University College of Medicine, \\ Department of Pediatrics, Philadelphia, \\ Pennsylvania \\ ${ }^{2}$ University of Wisconsin School of Medi- \\ cine and Public Health, Department of \\ Family Medicine, Madison, Wisconsin \\ ${ }^{3}$ Drexel University Dornsife School of \\ Public Health, Department of Commu- \\ nity Health and Prevention, Philadelphia, \\ Pennsylvania
}

Conflicts of interest: authors report none.

\section{CORRESPONDING AUTHOR}

Jeffrey P. Yaeger, MD, MPH St. Christopher's Hospital for Children 160 East Erie Avenue

Philadelphia, PA 19134

jeffrey.yaeger@drexelmed.edu

\begin{abstract}
PURPOSE Prior studies have evaluated factors predictive of inappropriate antibiotic prescription for upper respiratory tract infections (URIs). Community factors, however, have not been examined. The aim of this study was to evaluate the roles of patient, clinician, and community factors in predicting appropriate management of URIs in children.
\end{abstract}

METHODS We used a novel database exchange, linking electronic health record data with community statistics, to identify all patients aged 3 months to 18 years in whom URI was diagnosed in the period from 2007 to 2012. We followed the Healthcare Effectiveness Data and Information Set (HEDIS) quality measurement titled "Appropriate treatment for children with upper respiratory infection" to determine the rate of appropriate management of URIs. We then stratified data across individual and community characteristics and used multiple logistic regression modeling to identify variables that independently predicted antibiotic prescription.

RESULTS Of 20,581 patients, the overall rate for appropriate management for URI was $93.5 \%$. Family medicine clinicians $(A O R=1.5 ; 95 \% \mathrm{Cl} 1.31,1.71$; reference $=$ pediatric clinicians), urgent care clinicians $(A O R=2.23 ; 95 \% \mathrm{Cl} 1.93,2.57$; reference $=$ pediatric clinicians), patients aged 12 to 18 years $(A O R=1.44 ; 95 \%$ Cl 1.25, 1.67; reference $=$ age 3 months to 4 years), and patients of white race/ ethnicity (AOR $=1.83 ; 95 \% \mathrm{CI} 1.41,2.37$; reference $=$ black non-Hispanic) were independently predictive of antibiotic prescription. No community factors were independently predictive of antibiotic prescription.

CONCLUSIONS Results correlate with prior studies in which non-pediatric clinicians and white race/ethnicity were predictive of antibiotic prescription, while association with older patient age has not been previously reported. Findings illustrate the promise of linking electronic health records with community data to evaluate health care disparities.

Ann Fam Med 2015;13:529-536. doi: 10.1370/afm.1856.

\section{INTRODUCTION}

U pper respiratory tract infections (URIs) are one of the most frequent acute reasons for children to seek medical attention, with 500 million cases each year accounting for more than $10 \%$ of all ambulatory and emergency department encounters. ${ }^{1}$ Because the vast majority of colds are attributed to viral processes, the use of antibiotics to treat URIs is not recommended. Although the frequency of antibiotic prescription for URIs seems to have been decreasing since the early 1990 s, $^{2}$ studies indicate that antibiotics are prescribed for colds up to $20 \%$ of the time. $^{3}$ The problems associated with the misuse of antibiotics in treating URIs in children are well established ${ }^{4}$ and include the development of drug resistant organisms, ${ }^{5}$ potential side effects/toxicities with no clinical benefit, and contribution to rising health care costs. ${ }^{6}$

The National Committee on Quality Assurance (NCQA), through its Healthcare Effectiveness Data and Information Set (HEDIS), has devel- 
oped the "Appropriate treatment for children with upper respiratory infection" measure to estimate the annual percentage of US children who were diagnosed with a URI and were not prescribed an antibiotic. ${ }^{7}$ The HEDIS measure is helpful in giving a broad national snapshot of antibiotic overuse, but its utility is limited. Individual characteristics such as sex, age, race/ ethnicity, home location, and socioeconomic status are important factors that influence health but are not included in the HEDIS data. ${ }^{8}$

Prior studies have examined patient and clinician factors associated with inappropriate prescription of antibiotics for URIs in children. Independent predictors include age 5 to 11 years, ${ }^{9,10}$ being diagnosed with bronchitis, ${ }^{9-12}$ and being seen by a nonpediatrician. ${ }^{9,10,13,14}$ When treated by the same clinician, black children were less likely to receive antibiotics. ${ }^{15}$ Emergency medicine physicians were more likely to give antibiotics for URI than primary care physicians. ${ }^{13}$ Clinicians were also more likely to give antibiotics inappropriately to all children of low socioeconomic status ${ }^{16}$ and to those with private insurance. ${ }^{17}$ Clinicians who are further removed from medical school give antibiotics more frequently. ${ }^{18,19}$ Antibiotics are prescribed more often for URIs when they are perceived by the clinician as being expected by the parents. ${ }^{20,21}$ Social concordance between clinician and patient/family is an important factor in predicting inappropriate prescription of antibiotics. ${ }^{22}$ Some studies have relied on information collected through the National (Hospital) Ambulatory Medical Care Survey, ${ }^{4,9,10,12}$ whereas others have used claims data, ${ }_{1}^{19,23}$ or surveys. ${ }^{24}$

As the ecologic health systems model describes, not only are individual biologic factors and behaviors important, but larger societal constructs, such as family, environment, and community characteristics, are also major determinants of health. ${ }^{25}$ For example, the urbanization status of a patient's home community, ${ }^{26}$ the socioeconomic status of the community where the clinic is located ${ }_{1}{ }^{27}$ and the clinician's perception of patient education level ${ }^{28}$ can influence the clinician's management for several medical conditions. None of these community characteristics are captured by the national HEDIS measure.

To our knowledge, no previous studies have used electronic health record (EHR) data to evaluate antibiotic prescription for children with URIs. At the same time, the influence of community characteristics in predicting the management for pediatric URIs is unclear. Both of these issues represent gaps in our understanding of the management of pediatric URIs.

Our goals for this study were twofold. First, we aimed to apply the HEDIS protocol to EHR data to estimate the frequency of appropriate management of children with URI and compare these findings with national data. Second, we sought to identify previously undescribed patient, clinician, and particularly community characteristics that could predict antibiotic prescription for URI.

\section{METHODS}

The University of Wisconsin Electronic Health Record - Public Health Information Exchange (UW eHealth - PHINEX) data set was used to identify eligible pediatric patients. PHINEX is a large clinical data exchange established between UW Health (University of Wisconsin Departments of Family Medicine, Internal Medicine, and Pediatrics) and the Wisconsin State Division of Public Health. Patients seen through these primary care departments also have their data included from all other UW Health episodes of care, such as hospitalizations, emergency department visits, and urgent care visits. Specifically designed to statistically represent the ecologic health systems model, PHINEX examines health and disease within the patient's biologic, psychosocioeconomic, environmental, and community context. ${ }^{29}$ It merges EHR data with public databases of geographic, environmental, socioeconomic, and demographic profiles to create a comprehensive source for investigation of infectious, acute, chronic, injury-related, occupational, and environmental health outcomes and risk factors. It then employs multivariate analysis and data mining tools to model variables that could predict disease and health quality at the census block group level.

\section{Study Population}

The extract comprises data from 2007 to 2012 from 42 University of Wisconsin Clinics throughout the state, with over 180 faculty and 100 resident physicians (Figure 1). The demographic characteristics of the PHINEX sample population closely resembles that of the state of Wisconsin. ${ }^{29}$ Over 21 million encounters, including, among others, clinician, nurse, laboratory, and radiology visits, and approximately 500,000 individuals are included. PHINEX is able to extract all clinician orders without reliance upon pharmacy data, so information is available regarding all prescriptions ordered, not only those filled at the pharmacy. Approximately 6,000 variables are available at the census block group level, including community demographics, street data, socioeconomic segmentation, and community education. ${ }^{29}$

The data exchange and this study were both approved by the University of Wisconsin Institutional Review Board. All data are de-identified and comply with the HIPAA privacy rules. 


\section{Measures}

For phase 1 of this study, the protocol defined in the HEDIS 2011 measure, "Appropriate treatment for children with upper respiratory infection" was duplicated using the PHINEX data. ${ }^{30}$ This yielded a population aged 3 months to 18 years of age who were seen in an ambulatory clinic and were diagnosed with only URI. Emergency department visits were excluded because information was not available. To comply with the HEDIS protocol, we identified children with visits where a diagnosis of nasopharyngitis or URI alone was documented; that is, International Classification of Diseases, 9th Revision, Clinical Modification (ICD-9 CM) code 460 or code 465 , respectively. All encounters where an antibiotic was prescribed (as defined by HEDIS Table CWP-C) ${ }^{30}$ in the previous 30 days were excluded, as were encounters with more than 1 diagnosis and encounters in which any of a defined set of competing diagnoses was made on or within 3 days after the episode. ${ }^{30}$ Because the HEDIS protocol uses the terminology "Appropriate Treatment" when referring to those children who were not prescribed antibiotics, this terminology will be used throughout the text. The protocol does not give information about other management modalities such as cold medicine.
PHINEX measurements were then compared with national HEDIS data.

For phase 2, variables of interest included clinician specialty, patient age, patient sex, patient race/ethnicity (as provided in the EHR), comorbidities, health insurance type, community-level economic hardship, urbanization level of home location, and community education level. Comorbid conditions included neoplasm and asthma, which affected $3.1 \%$ and $16 \%$ of the population, respectively. Other comorbid conditions that were considered included chronic lung disease, congenital heart disease, immunodeficiency, cystic fibrosis, and diabetes; these were excluded because each disease occurred in less than $2 \%$ of the population. Six indicators, including crowded housing, poverty, unemployment, education, dependency, and income level, were used to describe the social and economic condition of an individual's community condition at the census block group level; together, they are referred to as the economic hardship index. ${ }^{30}$ ${ }^{32}$ Location of the patient's home was categorized as rural, suburban, or urban, derived from segmentation methodology and information regarding population density, size of city, and location relative to a metropolitan area. ${ }^{33,34}$

\section{Figure 1. Geographic distribution of patients in the PHINEX database exchange.}

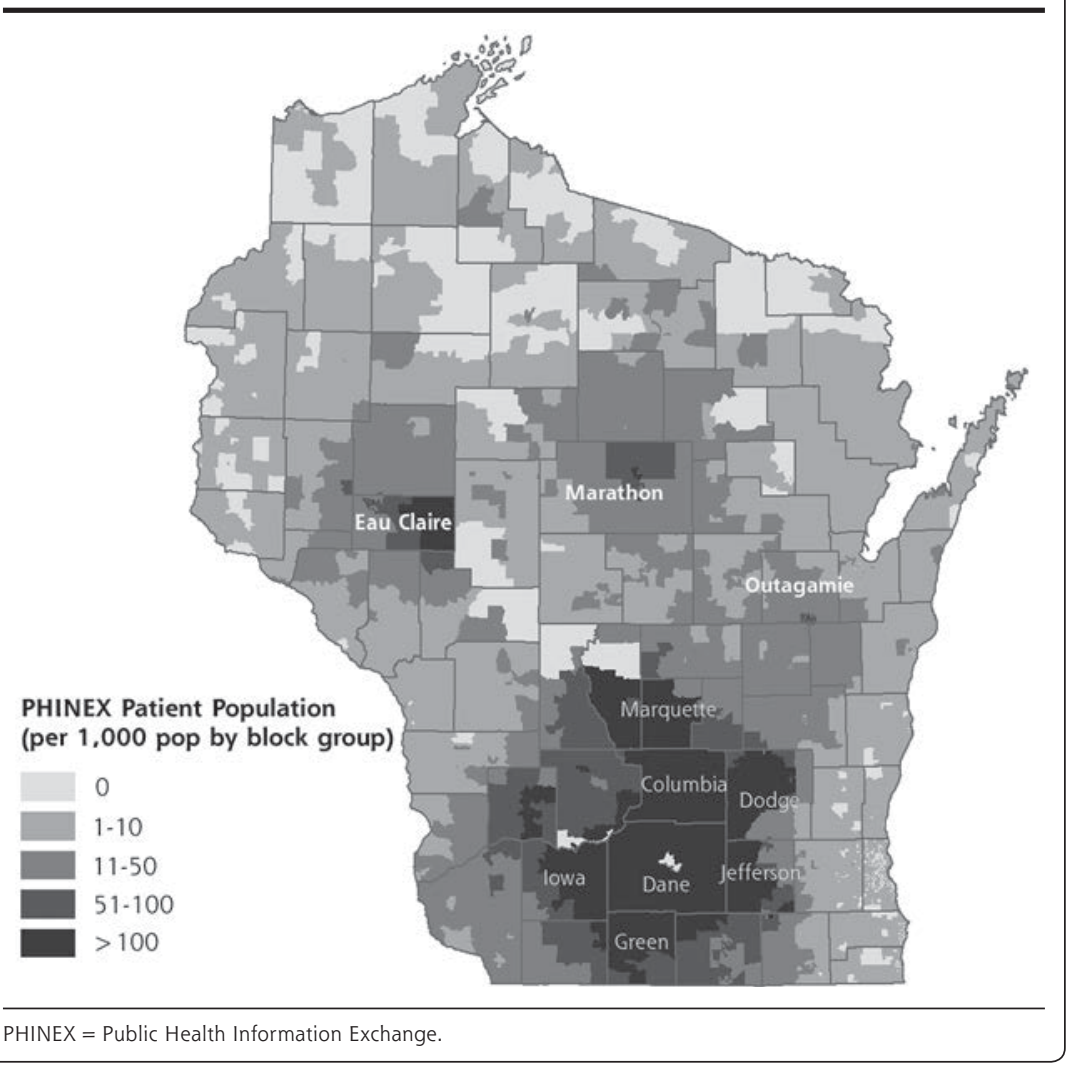

\section{Statistical Analysis}

For phase 1, cumulative data from all 6 years (2007 to 2012) were used. To compare PHINEX data with national HEDIS data, crude percentages of appropriate use of antibiotics were calculated for each year. For phase 2, we calculated crude odds ratios for each variable. Then an automated step-wise logistic regression selection was performed to develop a model capable of predicting independent factors involved in the prescription of antibiotics for URI. ${ }^{35}$ This model was used to calculate odds ratios, $95 \%$ confidence intervals (CIs), and $P$ values for these independently predictive variables. The level of significance was defined as $P$ value less than .05 and $\mathrm{CI}$ that did not include 1 .

\section{RESULTS}

In all, 20,581 patients met inclusion criteria. About $60 \%$ were aged between 3 months and 4 
years old, almost one-half were seen in pediatric clinics, one-half were male, about $80 \%$ were white, the majority had no comorbidities, and most had commercial health insurance (Table 1). Most patients were from suburban neighborhoods with moderate levels of economic hardship and high community education level (Table 1).

Table 1. Demographic Characteristics of Study Population

\begin{tabular}{|c|c|c|}
\hline Characteristic & $\begin{array}{c}\text { Patients } \\
\text { With URI } \\
N=20,581\end{array}$ & $\begin{array}{c}\text { Patients } \\
\text { Who Received } \\
\text { Antibiotics } \\
n=1,343\end{array}$ \\
\hline \multicolumn{3}{|c|}{ Year of encounter, No. (\%) } \\
\hline 2007 & $3,071(15)$ & $193(6.3)$ \\
\hline 2008 & $3,244(16)$ & $223(6.9)$ \\
\hline 2009 & $4,336(21)$ & $279(6.4)$ \\
\hline 2010 & $3,367(16)$ & $204(6.1)$ \\
\hline 2011 & $3,369(16)$ & $238(7.1)$ \\
\hline 2012 & $3,194(16)$ & $206(6.4)$ \\
\hline \multicolumn{3}{|l|}{ Age, No. (\%) } \\
\hline 3 mo to $4 y$ & $12,314(60)$ & $707(5.7)$ \\
\hline $5-11$ y & $5,036(24)$ & $347(6.9)$ \\
\hline $12-18$ y & $3,231(16)$ & $289(8.9)$ \\
\hline \multicolumn{3}{|l|}{ Sex, No. (\%) } \\
\hline Female & $10,217(50)$ & $681(6.7)$ \\
\hline Male & $10,364(50)$ & $662(6.4)$ \\
\hline \multicolumn{3}{|l|}{ Race, ethnicity, No. (\%) } \\
\hline White (non-Hispanic) & $16,007(80)$ & $1,112(6.9)$ \\
\hline Other (non-Hispanic) & $1,094(5)$ & $52(4.8)$ \\
\hline Hispanic & $1,385(7)$ & $76(5.5)$ \\
\hline Black (non-Hispanic) & $1,506(8)$ & $66(4.4)$ \\
\hline \multicolumn{3}{|l|}{ Department, No. (\%) } \\
\hline Family medicine & $6,803(33)$ & $491(7.2)$ \\
\hline Urgent care & 3,987 (19) & $390(9.8)$ \\
\hline Pediatrics & $9,791(48)$ & $462(4.7)$ \\
\hline \multicolumn{3}{|l|}{ Comorbidities, No. (\%) } \\
\hline Yes & $4,730(23)$ & $344(6.5)$ \\
\hline No & $15,851(77)$ & $999(6.3)$ \\
\hline \multicolumn{3}{|c|}{ Health insurance, No. (\%) } \\
\hline Medicaid & $3,506(17)$ & $187(5.3)$ \\
\hline None & $2,901(14)$ & $270(9.3)$ \\
\hline Commercial & $14,174(69)$ & $886(6.3)$ \\
\hline \multicolumn{3}{|l|}{ Urbanization, No. (\%) } \\
\hline Suburban & $10,723(55)$ & $748(7.0)$ \\
\hline Rural & $3,072(16)$ & $213(6.9)$ \\
\hline Urban & $5,714(29)$ & $324(5.7)$ \\
\hline \multicolumn{3}{|c|}{$\begin{array}{l}\text { Community economic hardship index, } \\
\text { No. (\%) }\end{array}$} \\
\hline$<20$ & $1,724(9)$ & $115(6.7)$ \\
\hline 20 to $<30$ & $16,379(84)$ & $1,088(6.6)$ \\
\hline$\geq 30$ & $1,408(7)$ & $82(5.8)$ \\
\hline \multicolumn{3}{|c|}{$\begin{array}{l}\text { Community education level (percentage } \\
\text { with some college education), No. (\%) }\end{array}$} \\
\hline$<50 \%$ & $1,842(9)$ & $143(7.8)$ \\
\hline $50 \%$ to $<70 \%$ & $7,382(38)$ & $482(6.5)$ \\
\hline$\geq 70 \%$ & $10,285(53)$ & $660(6.4)$ \\
\hline
\end{tabular}

From 2007 to 2012, the percentage of children treated for URI without prescription of an antibiotic in the PHINEX data ranged from $92.9 \%$ to $93.9 \%$ (Figure 2). PHINEX consistently had higher scores than national HEDIS data for each year and insurance type (Figure 2).

Univariate analysis demonstrated that antibiotic prescription rates for URI were highest for encounters in urgent care settings and for patients of white race/ethnicity (Table 2). Crude odds ratios demonstrate that patients were more than 1.5 times as likely to receive antibiotics when seen in a family medicine clinic and more than 2 times as likely when seen in urgent care as when seen in pediatric clinics (Table 2); the absolute differences were $2.5 \%$ and $5.1 \%$, respectively (Table 1 ). Older children were more likely to receive antibiotics than children aged 3 months to 4 years (Table 2). Other factors that were associated with antibiotic prescription for URI in the univariate model included the presence of comorbidity, race/ethnicity, lack of health insurance, and rural or suburban home location as well as lower community education levels (Table 2). Medicaid insurance status was associated with fewer antibiotic prescriptions for URI than commercial insurance (Table 2).

Multiple logistic regression identified 3 independent predictors for prescription of antibiotics for an URI-clinician specialty, patient age, and race/ethnicity-with 4 effects of these variables reaching statistical significance (Figure 3). Patients seen in family medicine and urgent care clinics were 1.5 and 2.23 times as likely to be prescribed antibiotics as those seen in pediatric clinics, respectively. After controlling for other factors, adolescents and white (non-Hispanic) patients are 1.4 and 1.8 times as likely to be prescribed antibiotics, respectively.

\section{DISCUSSION}

The overall rates of appropriate management of URI in children in the PHINEX data set ranged from $92.9 \%$ (2011) to $93.9 \%$ (2010). These rates are higher than in the national HEDIS rate, which ranged from $83.5 \%$ in 2007 to $87.2 \%$ in $2010 .{ }^{2}$ Considering that we did not directly observe coding behavior, it is difficult to determine the reason for this discrepancy. Contributing factors, however, may include the facts that most patients were seen in pediatric clinics and that the study 
Figure 2. Percentages of patients receiving appropriate treatment of URI, 2007-2012.

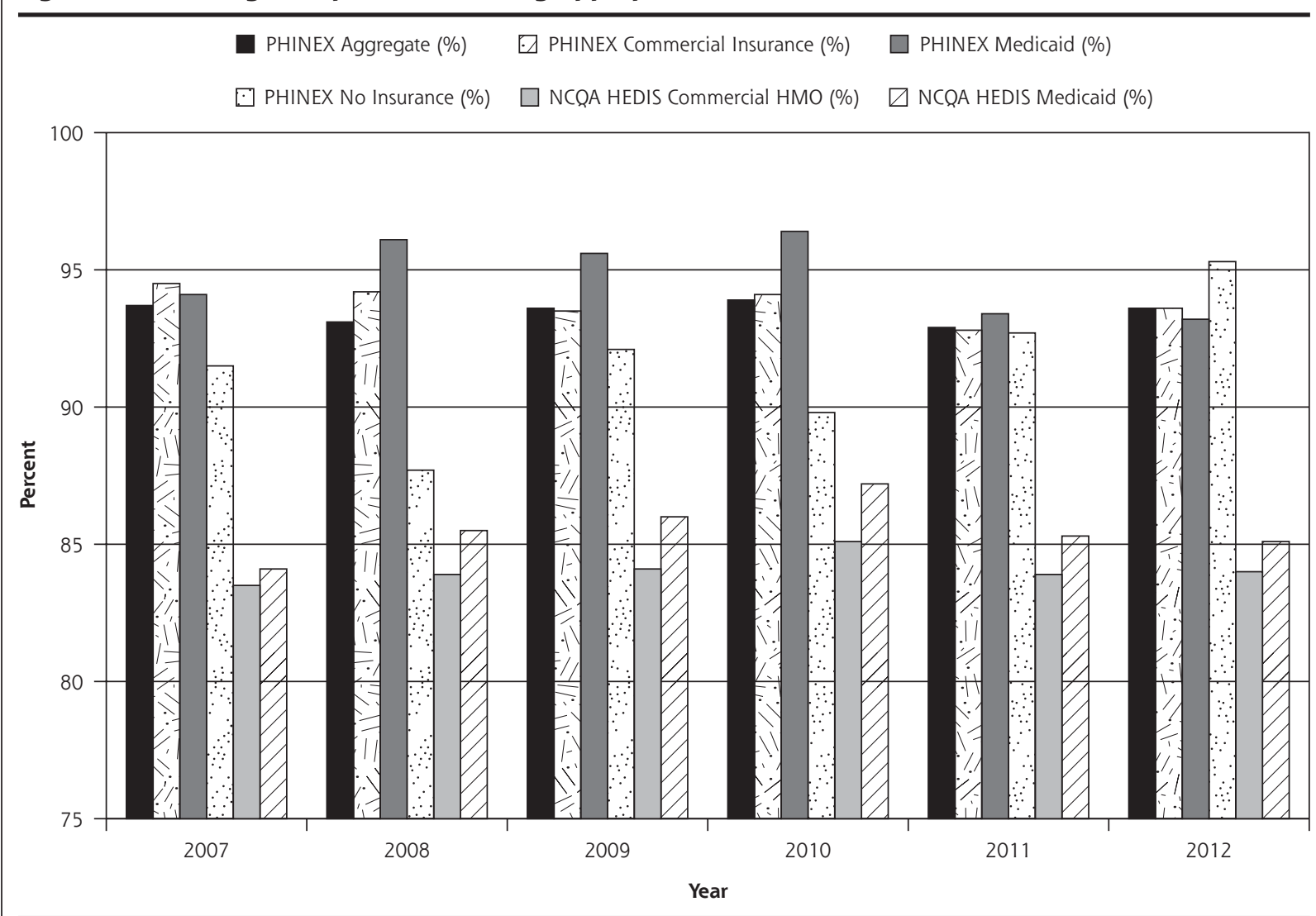

HEDIS = Healthcare Effectiveness Data and Information Set; NCQA = National Committee on Quality Assurance; PHINEX = Public Health Information Exchange; URI = upper respiratory tract infection.

NCQA data from National Committee for Quality Assurance. Improving Quality and Patient Experience: The State of Health Care Quality 2013. Washington, DC: National Committee for Quality Assurance; 2013.

occurred shortly after an extensive initiative to educate clinicians about antibiotic stewardship. ${ }^{36}$

This study did not identify any community factors that were independently predictive of inappropriate antibiotic prescription for URI. There may be several explanations for this. First, community factors may not be independently associated with antibiotic prescription for URIs. Second, the community factors evaluated in this study may be integrally linked with other characteristics, such as race/ethnicity or insurance status, diluting their overall effect. Third, although demographically representative of Wisconsin, the sample consisted of a population of limited racial, ethnic and economic diversity. Fourth, all patients were treated at clinics belonging to a large university network, thus reducing the level of variance in clinic-level and possibly patient-level variables. This highlights the need to continue studying disparities so that community factors on health care quality and equity can be understood more thoroughly.

In the univariate analysis, clinician specialty, patient age, patient race/ethnicity, presence of comorbidi- ties, health insurance status, rural home location, and low community education level were all found to be statistically predictive of prescription of antibiotics. Only clinician specialty, patient age, and patient race/ ethnicity, however, independently predicted antibiotic prescription in the multivariate model. This may be because these different predictors were highly colinear or because the association was confounded by other significant predictors in the model or both. Still, 2 community-level factors - urbanization and community education level—were at least initially predictive of prescription of antibiotics. Perhaps in other populations, these variables will show a stronger association.

As illustrated by the multivariate analysis, prescription of antibiotics varies widely depending on the population demographics. Clinician specialty, patient age, and patient race/ethnicity were the 3 independent predictors of antibiotic prescription for URI, confirming previous studies. ${ }^{9} 10,13,14,16,37$ Considering that this appears to be the first time EHR data were used to evaluate URI management in children, confirmation of 
prior findings strongly supports the validity of using EHRs to evaluate pediatric URIs.

Clinician specialty showed the strongest association, with non-pediatric clinicians prescribing antibiotics more frequently. It may seem that this is simply a clinician issue, as the clinician ultimately decides to give antibiotics. However, this may oversimplify the complex nature of these encounters. There is more to

\begin{tabular}{|c|c|c|}
\hline Characteristic & $\begin{array}{l}\text { Crude OR } \\
(95 \% \mathrm{CI})\end{array}$ & $P$ Value \\
\hline \multicolumn{3}{|l|}{ Year } \\
\hline 2007 & $0.98(0.81-1.18)$ & .79 \\
\hline 2008 & $1.07(0.9-1.29)$ & .45 \\
\hline 2009 & 1.00 (Reference) & -- \\
\hline 2010 & $0.94(0.78-1.13)$ & .5 \\
\hline 2011 & $1.11(0.92-1.32)$ & .27 \\
\hline 2012 & $1.00(0.83-1.21)$ & .98 \\
\hline \multicolumn{3}{|l|}{ Department } \\
\hline Family medicine & $1.57(1.38-1.79)$ & $<.001$ \\
\hline Urgent care & $2.19(1.9-2.52)$ & $<.001$ \\
\hline Pediatrics & 1.00 (Reference) & -- \\
\hline \multicolumn{3}{|l|}{ Age } \\
\hline 5-11 y & $1.22(1.1-1.39)$ & .004 \\
\hline $12-18$ y & $1.61(1.4-1.86)$ & $<.001$ \\
\hline 3 mo to $4 y$ & 1.00 (Reference) & -- \\
\hline \multicolumn{3}{|l|}{ Sex } \\
\hline Female & $1.05(0.94-1.17)$ & .42 \\
\hline Male & 1.00 (Reference) & -- \\
\hline \multicolumn{3}{|l|}{ Race/ethnicity } \\
\hline White (Non-Hispanic) & $1.63(1.26-2.1)$ & $<.001$ \\
\hline Other (Non-Hispanic) & $1.09(0.75-1.58)$ & .65 \\
\hline Hispanic & $1.27(0.9-1.78)$ & .17 \\
\hline Black (Non-Hispanic) & 1.00 (Reference) & -- \\
\hline \multicolumn{3}{|l|}{ Comorbidities } \\
\hline Yes & $1.17(1.03-1.32)$ & .02 \\
\hline No & 1.00 (Reference) & -- \\
\hline \multicolumn{3}{|l|}{ Health insurance } \\
\hline Medicaid & $0.84(0.72-0.99)$ & .04 \\
\hline None & $1.54(1.34-1.78)$ & $<.001$ \\
\hline Commercial & 1.00 (Reference) & -- \\
\hline \multicolumn{3}{|l|}{ Urbanization } \\
\hline Suburban & $1.25(1.09-1.43)$ & .001 \\
\hline Rural & $1.24(1.04-1.48)$ & .02 \\
\hline Urban & 1.00 (Reference) & -- \\
\hline \multicolumn{3}{|l|}{$\begin{array}{l}\text { Community economic } \\
\text { hardship index }\end{array}$} \\
\hline 20 to $<30$ & $1.00(0.82-1.22)$ & .96 \\
\hline$\geq 30$ & $0.87(0.65-1.16)$ & .33 \\
\hline$<20$ & 1.00 (Reference) & -- \\
\hline \multicolumn{3}{|c|}{$\begin{array}{l}\text { Community education level } \\
\text { (percentage with some col- } \\
\text { lege education) }\end{array}$} \\
\hline$<50 \%$ & $1.23(1.02-1.48)$ & .03 \\
\hline 50 to $<70 \%$ & $1.02(0.9-1.15)$ & .76 \\
\hline$\geq 70 \%$ & 1.00 (Reference) & -- \\
\hline
\end{tabular}

the patient/family-physician interaction than merely the clinician examining the patient and providing a treatment. ${ }^{20-22,28}$ Findings suggest that broader issues of patient/family self-efficacy and effective interactions persist in the health care system.

Adolescent age group was an independent predictor of antibiotic prescription. From our review of the literature, this has not been previously demonstrated Perhaps older adolescents can verbalize their symptoms and preferences more effectively than younger children, conveying an expectation of antibiotics. Alternatively, clinicians may perceive adolescents as less likely to follow up if symptoms worsen and may over-treat individuals in this age group. The association may also suggest perception of cost opportunities (eg, school days lost) that may be more important for an adolescent population than for younger children.

These findings contrast with the disparities found for other outcomes. Typically, health care disparities are associated with suboptimal care for racial/ethnic minorities and those with public insurance. In this instance, however, white children are treated inappropriately for URI almost twice as often as black children. These data indicate that black children and children seen in non-pediatric clinics, while treated appropriately, may be receiving disparate care.

Clinicians may give antibiotics for URIs for a multitude of reasons. First, despite extensive educational initiatives, some clinicians may not know better. Second, they may perceive that it is more important to accommodate the patient's or family's immediate requests for antibiotics in the hopes of building longer term continuity than to risk appearing uncompassionate and disenfranchising the family from further medical care. Third, clinicians may be unwilling or unable to adequately counsel families on the problems with giving antibiotics for URIs. Lastly, as demonstrated in studies of acute otitis media, this disparity may stem from race-based differences in practice patterns. ${ }^{38,39}$

Linking EHRs with community and public health data may offer new insights into these issues. Provision of broader clinician education regarding judicious antibiotic use should continue. Other actions could include linking reimbursement rates more closely with stratified HEDIS measures and continuing to develop a diverse pool of clinicians who can effectively serve the needs of a diverse population. But first, to identify and minimize health care disparities, epidemiologic data and HEDIS measures need to be stratified by demographic characteristics.

\section{Limitations}

This study has several limitations. First, all encounters were in academic clinics, and emergency department 


\section{Figure 3. Results of multivariate analysis.}

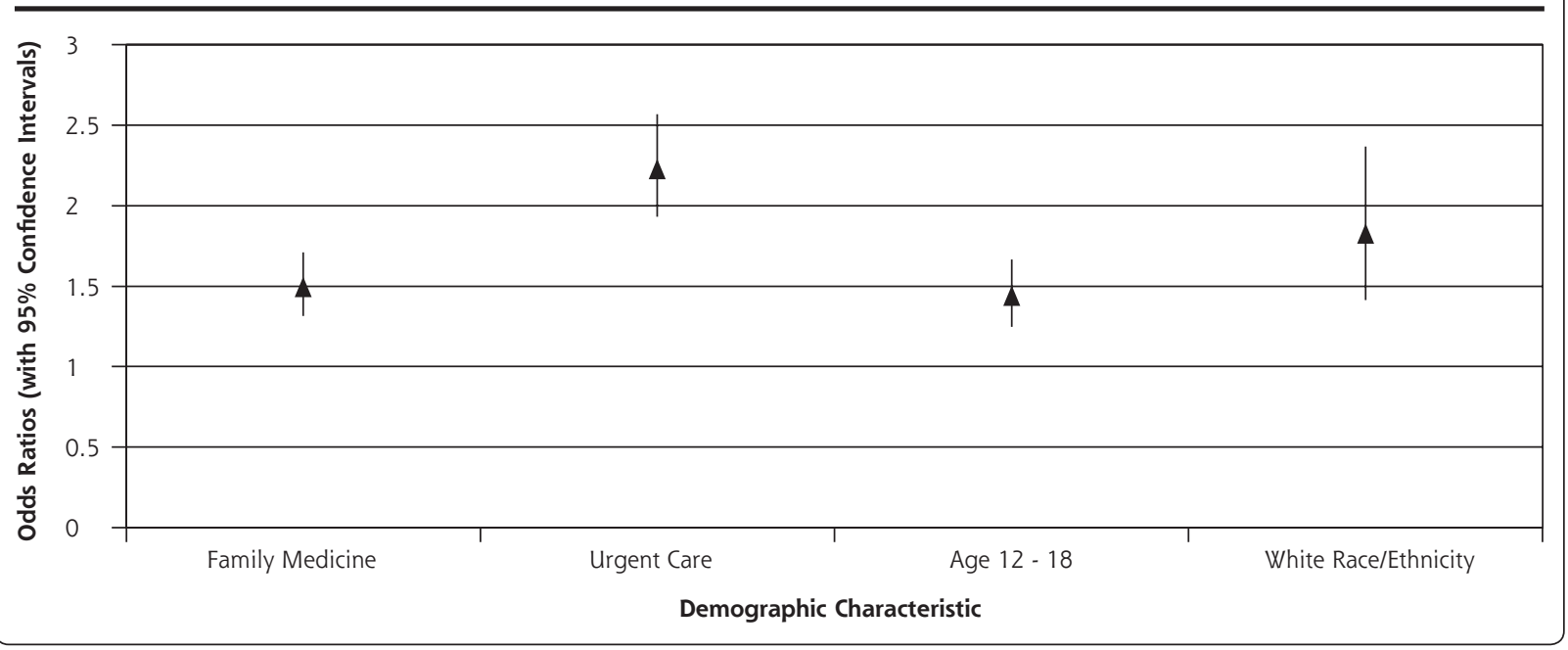

visits were excluded. Because PHINEX represents only 1 health system, it is possible that individuals may receive care at outside facilities that would not be captured in this data set. The study population is of limited racial/ethnic diversity, so it is unclear how findings may relate to other settings. More than $80 \%$ of patients were classified with moderate levels of economic hardship, possibly making it more difficult to detect economic hardship index as a predictor. Also, because we replicated the HEDIS protocol, only diagnostic codes for URI were used, and we did so without directly observing coding behavior. The rates of other infectious and respiratory processes such as acute otitis media and acute sinusitis in this population are unclear. Clinicians may have shifted their diagnoses to justify the provision of antibiotics, differentially affecting results. Future studies should explore this issue.

\section{CONCLUSIONS}

This is the first study to use EHR and community data to simultaneously evaluate the patient, clinician, and community factors affecting the management of pediatric URIs. White children, adolescents, and children seen in non-pediatric clinics are at increased risk of receiving antibiotics for URI, highlighting the importance of stratifying data across demographic characteristics. Replication of previous findings supports the use and validity of EHR data linked to public health information to evaluate antibiotic prescription in children. Adolescent age should be studied in other populations to confirm our findings. While community characteristics were not independently predictive in our study, they may be important in other populations or conditions.
In the future, PHINEX data could be used to evaluate other characteristics that may be associated with antibiotic administration for URIs in children, including time elapsed since residency training graduation, social concordance between clinician and patient, and time of day of encounter. Linking EHR data with public health information may have implications in addressing health care disparities for other outcomes as EHRs become more widely available. Further research should expand this data exchange to a more diverse population and compare individual neighborhoods through geospatial technology to identify local differences in health care provision and outcomes.

To read or post commentaries in response to this article, see it online at http://www.annfammed.org/content/13/6/529.

Key words: information management; informatics; electronic health records; public health; health care delivery; health services research; social conditions; infants; children; adolescents

Submitted March 27, 2015; submitted, revised, July 15, 2015; accepted August 3, 2015.

Funding support: The work presented here was carried out while Dr Yaeger was a Primary Care Research Fellow supported by a National Research Service Award (T32HP10010) from the Health Resources and Services Administration to the University Of Wisconsin Department Of Family Medicine.

Previous presentations: The data from this research were presented as an abstract at the Pediatric Academic Society Conference; May 3-6, 2014; Vancouver, British Columbia.

Acknowledgments: The authors would like to thank Aman Tandias, MS, whose expertise in data mining and statistical modeling were instrumental in performing this study. He did not receive additional compensation for his contributions. 


\section{References}

1. Linder JA. Improving care for acute respiratory infections: better systems, not better microbiology. Clin Infect Dis. 2007;45(9):1189-1191.

2. Grijalva CG, Nuorti JP, Griffin MR. Antibiotic prescription rates for acute respiratory tract infections in US ambulatory settings. JAMA. 2009;302(7):758-766.

3. McCaig LF, Besser RE, Hughes JM. Trends in antimicrobial prescribing rates for children and adolescents. JAMA. 2002;287(23):3096-3102.

4. Centers for Disease Control and Prevention (CDC). Office-related antibiotic prescribing for persons aged $\mathrm{f} 14$ years - United States, 1993-1994 to 2007-2008. MMWR Morb Mortal Wkly Rep. 2011; 60(34):1153-1156.

5. Dowell SF, Marcy SM, Phillips WR, Gerber MA, Schwartz B. Principles of judicious use of antimicrobial agents for pediatric upper respiratory tract infections. Pediatrics. 1998;101(Suppl 1):163-165.

6. Fendrick AM, Monto AS, Nightengale B, Sarnes M. The economic burden of non-influenza-related viral respiratory tract infection in the United States. Arch Intern Med. 2003;163(4):487-494.

7. National Committee for Quality Assurance. Improving Quality and Patient Experience: The State of Health Care Quality 2013. Washington, DC: National Committee for Quality Assurance; 2013.

8. Cheung CR, Gray JA. Unwarranted variation in health care for children and young people. Arch Dis Child. 2013;98(1):60-65.

9. Nash DR, Harman J, Wald ER, Kelleher KJ. Antibiotic prescribing by primary care physicians for children with upper respiratory tract infections. Arch Pediatr Adolesc Med. 2002;156(11):1114-1119.

10. Nyquist AC, Gonzales R, Steiner JF, Sande MA. Antibiotic prescribing for children with colds, upper respiratory tract infections, and bronchitis. JAMA. 1998;279(11):875-877.

11. Linder JA, Singer DE, Stafford RS. Association between antibiotic prescribing and visit duration in adults with upper respiratory tract infections. Clin Ther. 2003;25(9):2419-2430.

12. Mainous AG III, Hueston WJ, Davis MP, Pearson WS. Trends in antimicrobial prescribing for bronchitis and upper respiratory infections among adults and children. Am J Public Health. 2003;93(11): 1910-1914.

13. Nadeem Ahmed M, Muyot MM, Begum S, Smith P, Little C, Windemuller FJ. Antibiotic prescription pattern for viral respiratory illness in emergency room and ambulatory care settings. Clin Pediatr (Phila). 2010;49(6):542-547.

14. Schwartz B, Mainous AG III, Marcy SM. Why do physicians prescribe antibiotics for children with upper respiratory tract infections? JAMA. 1998;279(11):881-882.

15. Gerber JS, Prasad PA, Localio AR, et al. Racial differences in antibiotic prescribing by primary care pediatricians. Pediatrics. 2013; 131(4):677-684.

16. Mangione-Smith R, Elliott MN, Stivers T, McDonald LL, Heritage J. Ruling out the need for antibiotics: are we sending the right message? Arch Pediatr Adolesc Med. 2006;160(9):945-952.

17. Hersh AL, Shapiro DJ, Pavia AT, Shah SS. Antibiotic prescribing in ambulatory pediatrics in the United States. Pediatrics. 2011;128(6): 1053-1061.

18. Stone S, Gonzales R, Maselli J, Lowenstein SR. Antibiotic prescribing for patients with colds, upper respiratory tract infections, and bronchitis: A national study of hospital-based emergency departments. Ann Emerg Med. 2000;36(4):320-327.

19. Mainous AG III, Hueston WJ, Love MM. Antibiotics for colds in children: who are the high prescribers? Arch Pediatr Adolesc Med. 1998;152(4):349-352.

20. Scott JG, Cohen D, DiCicco-Bloom B, Orzano AJ, Jaen CR, Crabtree $B F$. Antibiotic use in acute respiratory infections and the ways patients pressure physicians for a prescription. J Fam Pract. 2001; 50(10):853-858.
21. Mangione-Smith R, Elliott MN, Stivers T, McDonald L, Heritage J, McGlynn EA. Racial/ethnic variation in parent expectations for antibiotics: implications for public health campaigns. Pediatrics. 2004;113(5):e385-e394.

22. Thornton RLJ, Powe NR, Roter D, Cooper LA. Patient-physician social concordance, medical visit communication and patients' perceptions of health care quality. Patient Educ Couns. 2011;85(3):e201-e208.

23. Brown DW, Taylor R, Rogers A, Weiser R, Kelley M. Antibiotic prescriptions associated with outpatient visits for acute upper respiratory tract infections among adult Medicaid recipients in North Carolina. N C Med J. 2003;64(4):148-156.

24. Belongia EA, Naimi TS, Gale CM, Besser RE. Antibiotic use and upper respiratory infections: a survey of knowledge, attitudes, and experience in Wisconsin and Minnesota. Prev Med. 2002;34(3):346-352.

25. Fielding JE, Teutsch S, Breslow L. A Framework for Public Health in the United States. Public Health Rev. 2010;32(1):174-189.

26. Gonzales R, Steiner JF, Sande MA. Antibiotic prescribing for adults with colds, upper respiratory tract infections, and bronchitis by ambulatory care physicians. JAMA. 1997;278(11):901-904.

27. Gorin SS, Ashford AR, Lantigua R, et al. Intraurban influences on physician colorectal cancer screening practices. J Natl Med Assoc. 2007;99(12):1371-1380.

28. van Ryn M, Burgess D, Malat J, Griffin J. Physicians' perceptions of patients' social and behavioral characteristics and race disparities in treatment recommendations for men with coronary artery disease. Am J Public Health. 2006;96(2):351-357.

29. Guilbert TW, Arndt B, Temte J, et al. The theory and application of UW ehealth-PHINEX, a clinical electronic health record-public health information exchange. WMJ. 2012;111(3):124-133.

30. Appropriate Treatment for Children With Upper Respiratory Infection (URI). Washington, DC: National Committee on Quality Assurance; 2011.

31. Montiel LM, Nathan RP, Wright DJ. An Update on Urban Hardship. Albany, NY: The Nelson A. Rockefeller Institute of Government; 2004.

32. Premature Deaths from Heart Disease and Stroke in Los Angeles County: A Cities and Communities Health Report. Los Angeles, CA: County of Los Angeles Dept of Health Services; 2006.

33. Esri Business Analyst Desktop Premium. Redlands, CA: Esri. http:// www.esri.com/software/arcgis/extensions/businessanalyst/data-usprem.html. Accessed May 15, 2012.

34. Mitchell A. ESRI Guide to GIS Analysis, Volume 2: Spatial Measurements and Statistics. Redlands, CA: Esri Press; 2005.

35. Kleinbaum DG. Logistic Regression: A Self Learning Text. New York, NY: Springer; 2010.

36. Kiang KM, Kieke BA, Como-Sabetti K, Lynfield R, Besser RE, Belongia EA. Clinician knowledge and beliefs after statewide program to promote appropriate antimicrobial drug use. Emerg Infect Dis. 2005;11(6):904-911.

37. Vanderweil SG, Pelletier AJ, Hamedani AG, Gonzales R, Metlay JP, Camargo (A Jr. Declining antibiotic prescriptions for upper respiratory infections, 1993-2004. Acad Emerg Med. 2007;14(4):366-369.

38. Fleming-Dutra KE, Shapiro DJ, Hicks LA, Gerber JS, Hersh AL. Race, otitis media, and antibiotic selection. Pediatrics. 2014;134(6): 1059-1066.

39. Woods CR, Jones VF. Variation in antimicrobial prescribing for otitis media by race: different wrinkle in disparity? Pediatrics. 2014; 134(6):1204-1206. 